Check for updates

Cite this: RSC Adv., 2017, 7, 41552

Received 14th July 2017

Accepted 14th August 2017

DOI: 10.1039/c7ra07736j

rsc.li/rsc-advances

\section{Solvatochromism of bright carbon dots with tunable long-wavelength emission from green to red and their application as solid-state materials for warm WLEDs $\uparrow$}

\author{
Sai Lin, Chun Lin, Meiling He, Rongrong Yuan, Yijun Zhang, Yongqiang Zhou, \\ Weidong Xiang (iD) and Xiaojuan Liang*
}

Herein, we present a facile approach to produce long-wavelength emitting carbon dots (CDs). A shift of the fluorescence emission wavelength of CDs from $630 \mathrm{~nm}$ to $520 \mathrm{~nm}$ can be easily acquired. The assynthesized CDs exhibit concentration-dependent PL property as well as the "solvatochromism" phenomenon, which are rarely known in CDs. As the concentration or solvent polarity of the CDs increases, the photoluminescence $(\mathrm{PL})$ wavelength exhibits a red shift. More importantly, the QY of these long-wavelength CDs is as high as $52.4 \%$, which is considerably higher than that mentioned in other reports. Moreover, on addition of an acid $(\mathrm{HCl})$, the surface $\mathrm{H}^{+}$-functionalization on the CDs leads to strong quenching of fluorescence emission, which is almost reversible after the addition of a strong base $(\mathrm{NaOH})$. Then, $\mathrm{CDs}$ based solid-state composites were fabricated using a combination of polyvinyl butyral (PVB) and CDs. Finally, CDs/PVB composites were deposited on the $\mathrm{Ce}^{3+}: \mathrm{Y}_{3} \mathrm{Al}_{5} \mathrm{O}_{12}\left(\mathrm{Ce}^{3+}: \mathrm{YAG}\right)$ phosphor-in-glass (Ce-PiG) via a screen-printing technology. Warm white light-emitting diodes (WLEDs) were then fabricated using these materials and GaN chips. The results indicate that these CDs possess a potential for future applications in warm WLEDs.

\section{Introduction}

Carbon dots (CDs) exhibit excellent potential for applications in bioimaging, printing inks, photocatalysis, sensors and light emitting diodes (LED) owing to their remarkable properties. ${ }^{\mathbf{1 - 8}}$ Hence, innumerable raw materials and methods have been reported for the synthesis of CDs. In most of the studies, the photoluminescence (PL) emission spectra of CDs, with maxima in the blue-green region and their absorption curves that show gradual attenuation without significant absorption bands in the visible region, are reported. ${ }^{9-13}$ However, it is difficult to prepare long-wavelength (capable of single wavelength excitation) and multicolor emissive CDs, and this greatly hinders their development and practical applications. ${ }^{\mathbf{6 1 4}}$ Acquiring fluorescence emission across the entire visible spectrum has been admitted as a crux of all practical applications, especially for LED and bioimaging; hence there is a necessity to successfully synthesize long-wavelength luminescent CDs. For example, an indoor solid-state light correlated color temperature (CCT) lower than $4000 \mathrm{~K}$ and a color rendering index (CRI) higher than 80 are

College of Chemistry and Materials Engineering, Wenzhou University, Wenzhou 325035, China. E-mail: xiangweidong001@126.com

$\dagger$ Electronic supplementary information (ESI) available. See DOI: $10.1039 / \mathrm{c} 7 \mathrm{ra07736j}$ strongly desired. ${ }^{15,16}$ Pang et al. synthesized efficient red CDs (centered at $608 \mathrm{~nm}$, quantum yield (QY) of 1.8\%) through surface oxidation of large sized CDs. ${ }^{17}$ Yuan and co-worker developed multicolor CDs from blue to red with a QY up to $12 \%$ for red fluorescence. ${ }^{18}$ Lin et al. ${ }^{19}$ developed a solvothermal reaction method to synthesize long-wavelength CDs from $p$-phenylenediamine using ethanol as the solvent. However, these CDs should be separated by a silica gel column, which is tedious and time-consuming and a quantum yield (QY) only up to $26.1 \%$ is obtained. Therefore, there is an urgent need to develop a method to synthesize long-wavelength CDs with high $\mathrm{PL}$ and QY values.

White LEDs (WLEDs) have attracted considerable attention due to their remarkable capability of energy conservation. ${ }^{20}$ Nowadays, CDs have become a promising candidate of for the fabrication of WLEDs. In some cases, CDs based WLEDs are usually fabricated by combining blue CDs with yellow and red CDs or GaN chips with yellow CDs. ${ }^{21}$ However, CDs always undergo self-quenching in the solid state. Just like in organic molecules, this phenomenon was ascribed to excessive resonance energy transfer or direct $\pi-\pi$ interactions. ${ }^{18-23} \mathrm{Qu}$ et al. ${ }^{24}$ developed a high QY orange emissive CDs (46\% in solution), but it still quenched in the solid-state aggregates. Compared with solution, the solid state CDs exhibit outstanding properties. However, among all reported red-emissive CDs, the fabrication 
of efficient solid state CDs with red emission have been scarce. Therefore, the development of efficient and stable red-emissive solid state CDs will have a significant impact on the application of CDs.

In this study, we report the synthesis of new long-wavelength CDs via a solvothermal route using o-phenylenediamine/ $p$-phenylenediamine as a starting material and $N, N$-dimethyl formamide (DMF) as the solvent, which is carried out without any separation process. Clearly, the obtained CDs exhibit two unique characters: one is the concentration-dependent photoluminescence property and the other is the intense solventdependent property. This indicates that the obtained CDs can shift gradually from $520 \mathrm{~nm}$ to $630 \mathrm{~nm}$ with an increase in their concentration in solution or a change in solvent polarity. Moreover, to solve the issue of fluorescence quenching of CDs in solid state, PVB is chosen as the solid-state matrix of the assynthesized CDs. A series of tunable color, tunable thickness and tunable shape CDs based solid-state materials could be conveniently acquired. Finally, due to the controlled width of wavelength, its thickness and its compatibility with industrial processes, the screen-printing technology was adopted to fabricate CDs films (CF coated on Ce:YAG phosphor-in-glass (Ce:PiG) substrate (PiG-CF)). The results show that these CDs have a promising application as red color converter in solidstate warm WLEDs.

\section{Experimental}

\subsection{Synthesis of CDs}

The different color CDs were prepared by hydrothermal method; $p$-phenylenediamine or $o$-phenylenediamine was chosen as the $\mathrm{C}$ source and $\mathrm{N}$ source and DMF was used as the solvent. Different combinations of $p$-phenylenediamine and $o$-phenylenediamine result in diverse luminescence, as tabulated in Table 1 . Typically, $p$-phenylenediamine or $o$-phenylenediamine $(0.05 \mathrm{~g})$ were separately heated in $10 \mathrm{~mL} \mathrm{N,N}$ dimethyl formamide (DMF) solvent at $200{ }^{\circ} \mathrm{C}$ for 4 hours in an autoclave, and the obtained samples were denoted as P-CDs and O-CDs. Without tedious purification, the bright longwavelength CDs were obtained.

\subsection{Preparation of CDs-based on solid-state materials}

Herein, $1 \mathrm{~g}$ PVB powder was dissolved in $10 \mathrm{~mL}$ ethanol, and the $\mathrm{P}$-CDs and O-CDs were separately added into the solution under sonication for $5 \mathrm{~min}$ to form homogeneous solutions. Next, each of the hybrid homogenized solutions was poured into the matrix to form films (noted as PCF and OCF) or bulk materials at room temperature. Finally, CDs-based on solid-state material were acquired.

\subsection{Fabrication of warm WLEDs}

The PiG with a of diameter $10 \mathrm{~mm}$ and thickness of $0.6 \mathrm{~mm}$ was fabricated using Ce:YAG yellow phosphor (4 wt\%) and the $\mathrm{TeO}_{2}-\mathrm{ZnO}-\mathrm{Sb}_{2} \mathrm{O}_{3}-\mathrm{Al}_{2} \mathrm{O}_{3}-\mathrm{B}_{2} \mathrm{O}_{3}-\mathrm{Na}_{2} \mathrm{O}$ glass via a two-step meltquenching route, as previously reported by our group. ${ }^{19}$ To achieve the stacking configuration, a facile screen-printing technology was adopted to deposit the CDs embedded on PVB film onto the upper surface of the PiG plate, followed by drying at room temperature. Finally, the LED was fabricated using PiG-CF as the luminescent material and GaN chips.

\subsection{Characterization}

To study the microstructure of CDs and the distribution of particle size, transmission electron microscopy (TEM) and highresolution TEM (HRTEM) images were obtained using FEI Tecnai F20 operating at $200 \mathrm{kV}$. To study the surface functional chemical groups, the Fourier transform infrared (FTIR) spectra were obtained, in which each sample was ground with $\mathrm{KBr}$ powder, and measured using a Bruker Equinox 55 FTIR spectrometer from 500 to $4000 \mathrm{~cm}^{-1}$. Furthermore, to study the surface structures of the CDs, X-ray photoelectron spectroscopy (XPS) analysis was carried out using an AXI Ultra DLD spectrometer with monochromatic $\mathrm{Al} \mathrm{K} \alpha$ as the excitation source. UV-vis spectra were recorded with a UV-2450 spectrometer, in which each sample was placed in cuvettes $(1.0 \mathrm{~cm}$ path length). Fluorescence spectra and PL quantum yield (QY) were acquired using a Horiba Jobin Yvon Fluromax-4P spectrophotometer equipped with absolute QY measurement apparatus. The QY of the samples was tested using an integrating sphere. First, in order to minimize internal filter effects, CDs solution was diluted to an absorption intensity below 0.08 at the optimal excitation wavelength (O-CDs: $460 \mathrm{~nm}$; P-CDs: $520 \mathrm{~nm}$ ). Then, two $10 \mathrm{~mm}$ fluorescence cuvettes were separately used to fill equal volumes of CDs solution and pure solvent as a contrast. Subsequently, the CDs solution was placed in the integrating sphere and excited with monochromatic light at the optimal excitation wavelength. The fluorescence spectra were recorded in the ranges of $450-470 \mathrm{~nm} ; 480-650 \mathrm{~nm} ; 510-530 \mathrm{~nm}$ and $535-750 \mathrm{~nm}$. Similarly, the fluorescence spectra of pure solvent were recorded under identical conditions. Finally, fluorescence

Table 1 Synthesis of CDs by different combinations of $C$ source and their color

\begin{tabular}{|c|c|c|c|c|}
\hline Sample & Carbon source & Solvent & Color & Emission peak \\
\hline P-CDs & $p$-Phenylenediamine $(0.05 \mathrm{~g})$ & DMF & Orange & $610 \mathrm{~nm}$ \\
\hline P-CDs-0.01 & $p$-Phenylenediamine $(0.01 \mathrm{~g})$ & DMF & Orange & $580 \mathrm{~nm}$ \\
\hline O-CDs & $o$-Phenylenediamine $(0.05 \mathrm{~g})$ & $\mathrm{DMF}$ & Green & $550 \mathrm{~nm}$ \\
\hline O-CDs-0.01 & $o$-Phenylenediamine $(0.01 \mathrm{~g})$ & $\mathrm{DMF}$ & Green-yellow & $530 \mathrm{~nm}$ \\
\hline O-CDs- 0.005 & $o$-Phenylenediamine $(0.005 \mathrm{~g})$ & DMF & Yellow & $520 \mathrm{~nm}$ \\
\hline
\end{tabular}


software was used to calculate the QY according to the spectra of both sample and pure solvent. Time resolved PL lifetime measurements were performed using a time-correlated singlephoton counting (TCSPC) lifetime spectroscopy system with a picosecond-pulsed diode laser (EPL-400 nm) as the single wavelength excitation light source. Optical properties such as color rendering index (CRI), color coordinates (CIE), correlated color temperature (CCT) and luminous efficacy (LE) were evaluated employing an integrating sphere (PMS-50, Everfine, China) under a forward current of $20 \mathrm{~mA}$.

\section{Results and discussion}

The schematic for the synthesis of different CDs is depicted in Fig. 1a. Clear red and yellow solutions (P-CDs and O-CDs respectively) were obtained when they were dispersed in DMF (as shown in Fig. 1b), and yellow and orange colors under UV irradiation (365 nm, Fig. 1c) were observed. Then, the morphology and size of P-CDs and O-CDs were characterized by TEM. As presented in Fig. 1d-g, the two samples exhibit uniform nanoparticles with average sizes of about $5.5 \mathrm{~nm}$ (O-CDs) and $7.1 \mathrm{~nm}$ (P-CDs). The HRTEM images (inset in Fig. 1d and f) of an individual CD exhibit well-resolved lattice fringes with a typical $d$-spacing of $0.21 \mathrm{~nm}$, which corresponds to the $d$-spacing of graphene $\{1-100\}$ planes. ${ }^{24,25}$ Knowing the surface groups can help understand their luminescence mechanism, the FTIR and XPS analyses were performed to determine the surface elemental characteristics of O-CDs and P-CDs. The similar FTIR spectra obtained for the two samples revealed the existence of similar chemical compositions (Fig. 2a). Notably, $\mathbf{a}$
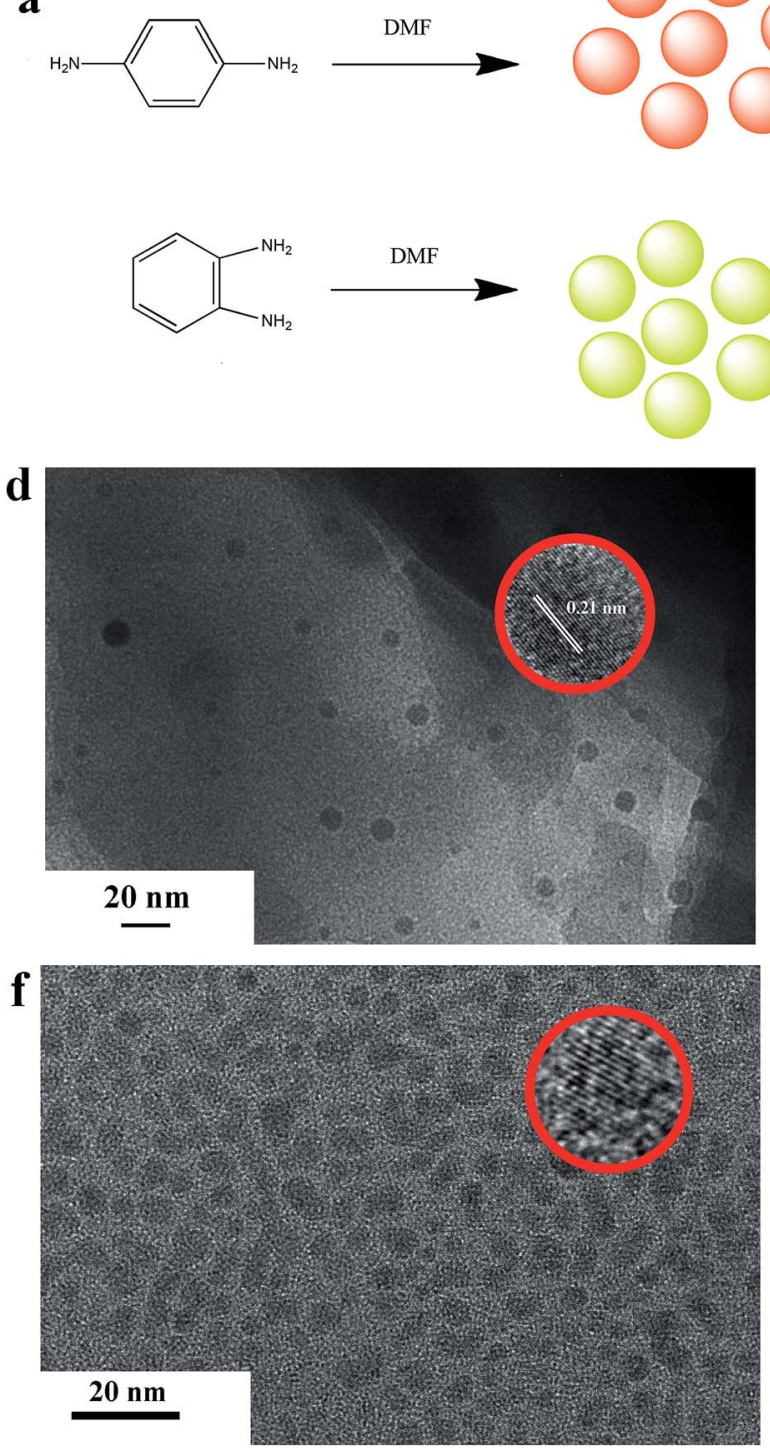

b
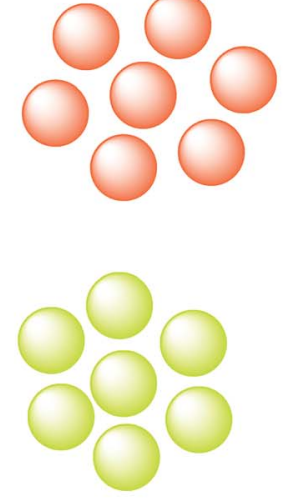

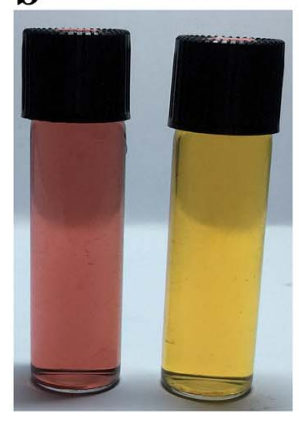

c

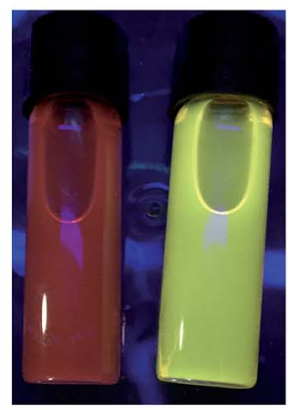

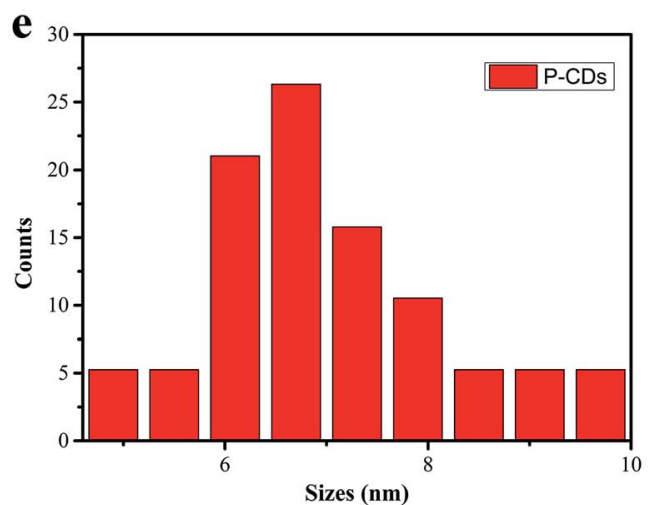

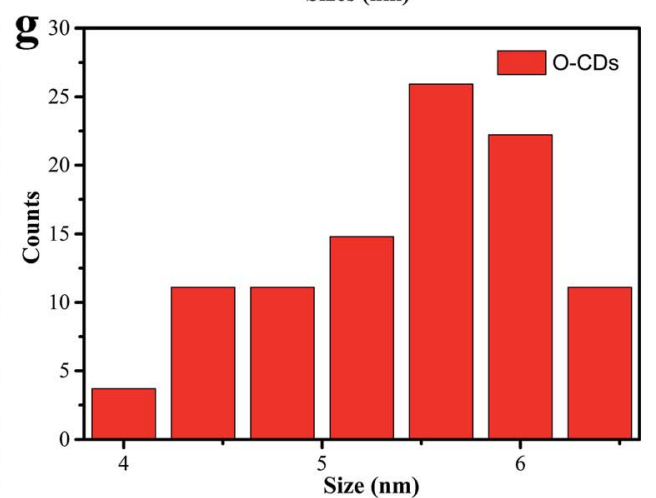

Fig. 1 (a) The schematic diagram of fabrication of long-wavelength CDs; (b) the images of P-CDs and O-CDs under sunlight and (c) under UV light; ( $d$ and f) TEM images of P-CDs and O-CDs, the insets are those of HRTEM; (e and $g$ ) the distribution of particles size. 
few new peaks emerged in comparison to the starting material. ${ }^{19}$ The broad brand at $3535 \mathrm{~cm}^{-1}$ is attributed to $\mathrm{O}-\mathrm{H} / \mathrm{N}-\mathrm{H}$; peaks emerging at $2934 \mathrm{~cm}^{-1}$ and $2862 \mathrm{~cm}^{-1}$ are ascribed to C$\mathrm{H}$, and the absorption bands at $1447 \mathrm{~cm}^{-1}$ and $1245 \mathrm{~cm}^{-1}$ are assigned to $\mathrm{C}=\mathrm{N}$ and aromatic ether, respectively. Large hydrophilic groups endow these CDs excellent dispersibility in various solvents, such as water, ethanol and DMF. The Fig. $2 b$ and $c$ exhibit the XPS full spectra of O-CDs and P-CDs, which demonstrate that the two CDs have the same elemental composition (C 1s $(\sim 285 \mathrm{eV}), \mathrm{N} 1 \mathrm{~s}(\sim 400 \mathrm{eV})$ and $\mathrm{O} 1 \mathrm{~s}$ $(\sim 532 \mathrm{eV}))$. It is obvious that $\mathrm{N}$ peak of $\mathrm{P}$-CDs is stronger than $\mathrm{O}-\mathrm{CD}$. The content of nitrogen, oxygen and carbon is tabulated in Table 2. In the high resolution spectra $\mathrm{C}$ 1s peak (Fig. $2 \mathrm{~d}$ and $\mathrm{g}$ ), the $\mathrm{C} 1 \mathrm{~s}$ band has been clearly resolved into three peaks at $284.7 \mathrm{eV}, 285.1 \mathrm{eV}$ and $286.7 \mathrm{eV}$, representing $\mathrm{C}=\mathrm{C} / \mathrm{C}-\mathrm{C}, \mathrm{C}-\mathrm{N}$ and $\mathrm{C}-\mathrm{O}$, respectively. The expanded spectra of $\mathrm{O} 1 \mathrm{~s}$ illustrates two peaks at $531.7 \mathrm{eV}$ and $533 \mathrm{eV}$, corresponding to $\mathrm{C}=\mathrm{O}$ and $\mathrm{C}-\mathrm{O}$, respectively (Fig. $2 \mathrm{e}$ and $\mathrm{h}$ ). The $\mathrm{N} 1 \mathrm{~s}$ is fitted into two peaks at $399.7 \mathrm{eV}$ and $401.8 \mathrm{eV}$, which are assigned to the pyrrole $\mathrm{N}$ and graphite $\mathrm{N}$, respectively (Fig. $2 \mathrm{f}$ and i) ${ }^{26}$

The UV-vis absorption and PL measurements are performed for determining optical properties of CDs. As shown in Fig. 3a
Table 2 XPS elemental analysis results of the O-CDs and P-CDs

\begin{tabular}{llll}
\hline Sample & $\mathrm{C}(\mathrm{mol} \%)$ & $\mathrm{O}(\mathrm{mol} \%)$ & $\mathrm{N}(\mathrm{mol} \%)$ \\
\hline O-CDs & $63.61 \%$ & $24.71 \%$ & $11.68 \%$ \\
P-CDs & $56.14 \%$ & $26.12 \%$ & $17.74 \%$
\end{tabular}

and c, UV-vis absorption spectra of O-CDs and P-CDs have a broad absorption that extends to the visible region, including two peaks at $250 \mathrm{~nm}$ and $298 \mathrm{~nm}$ in the UV region and a peak in the visible region. The peaks at $250 \mathrm{~nm}$ and $298 \mathrm{~nm}$ are ascribed to the $\pi-\pi^{*}$ transition of the aromatic $\mathrm{sp}^{2}$ domain and $\mathrm{C}=\mathrm{N}$, respectively. In comparison to $\mathrm{O}-\mathrm{CDs}$, the lower-energy and higher-energy absorption bands of the P-CDs exhibit a red-shift phenomenon, that is, $\lambda=298 \mathrm{~nm}$ to $318 \mathrm{~nm}$ and $\lambda=450 \mathrm{~nm}$ to $520 \mathrm{~nm}$, respectively. The peak at $520 \mathrm{~nm}$ is attributed to Mie scattering caused by CDs, which represents the typical absorption of an aromatic system and indicates an extended conjugation in the CDs structure. ${ }^{27}$ These red-shifts of the electronic absorption transitions demonstrate that the P-CDs contain smaller electronic band gaps than O-CDs, which are responsible for longer emission of P-CDs. Then, the PL properties of the
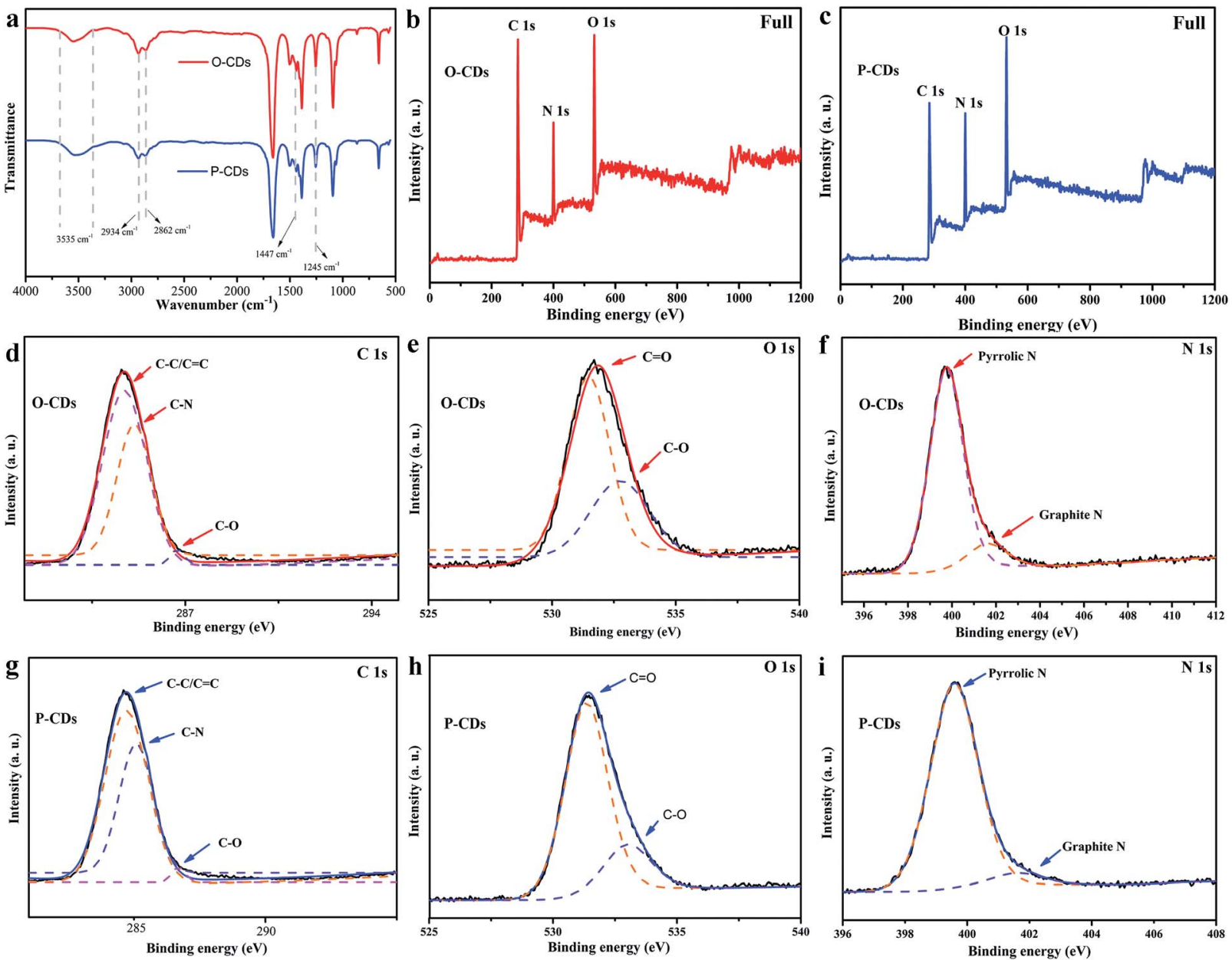

Fig. 2 (a) The FTIR spectra of samples; ( $b$ and c) XPS full survey of the O-CDs and P-CDs; the high resolution XPS spectra of (d and g) C 1s, (e and $h$ ) $O$ 1s and ( $f$ and i) $N$ 1s of two samples. 

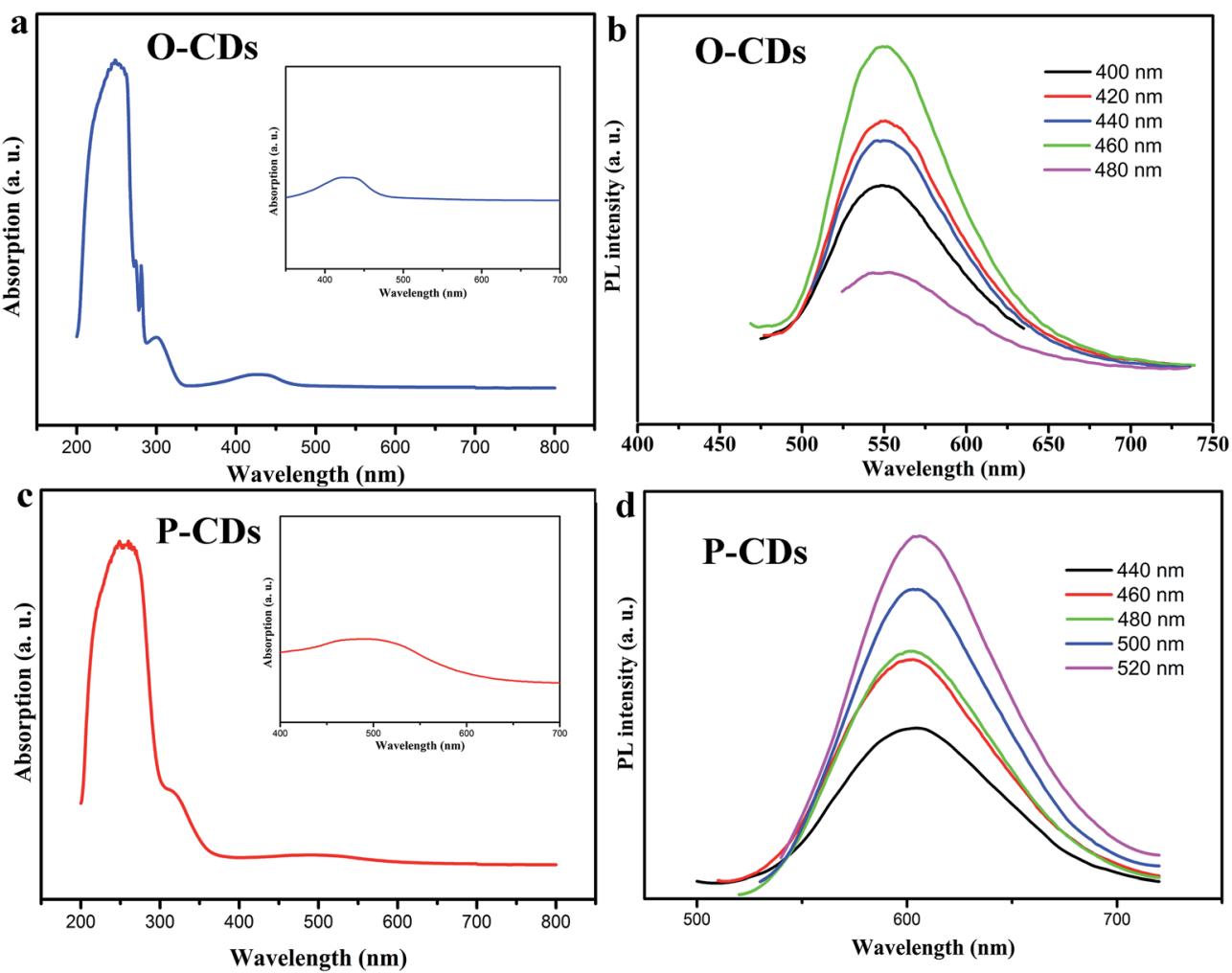

Fig. 3 (a and c) The UV-vis spectra of samples and the insets are UV-vis spectra in visible region; (b and d) the PL spectra of samples under different excitation wavelengths.

O-CDs and P-CDs were thoroughly investigated. The emission wavelengths of O-CDs and P-CDs are $\lambda=550 \mathrm{~nm}$ and $610 \mathrm{~nm}$ respectively (Fig. 3b and d). Even with an increase in excitation wavelength $\left(\lambda_{\mathrm{ex}}\right)$, the PL emission peaks remain nearly unshifted, which demonstrate the excitation-independent property of CDs. Further, the PLQY were measured by the absolute method. The QY of O-CDs and P-CDs solutions (DMF chromatographic grade) are up to $58.8 \%$ and $52.4 \%$, respectively (see Fig. S1 †). These QY values are higher than those of long wavelength emitting CDs as obtained in previous reports. ${ }^{19}$ The fluorescence decay is one of the intrinsic characteristics of an emittingstate. As a consequence, it is generally accepted that diverse emitting-states will exhibit different decay behaviors. As these two CDs exhibit very different PL spectra, P-CDs and O-CDs should also exhibit different PL decay behaviors. The PL decay of O-CDs and P-CDs are mono-exponential with lifetimes of 3.3 and 9.4 ns respectively (Fig. S2 $\dagger$ ). Furthermore, the concentration of the starting materials ( $p$-phenylenediamine or $o$-phenylenediamine) was investigated in detail. The corresponding PL spectra were measured, and are depicted in Fig. S3† and Table 1. When the amount of $p$-phenylenediamine was reduced from $0.05 \mathrm{~g}$ to $0.005 \mathrm{~g}$, the observed PL spectra blue shifts from $610 \mathrm{~nm}$ to $575 \mathrm{~nm}$. Analogously, on decreasing the amount of $o$-phenylenediamine from $0.05 \mathrm{~g}$ to $0.005 \mathrm{~g}$, the PL peak blue shifts gradually from $550 \mathrm{~nm}$ to $520 \mathrm{~nm}$. For each concentration type, different but strong emission peaks through small PL shifts with varying excitation are observed in low concentration samples (Fig. S3a†), which are similar to previous reports. ${ }^{28}$ It is believed that the uniform dispersion of the CDs in a solvent at high concentrations could be unstable because of the extremely short distance between individual CDs and its high surface energy, which eventually leads to aggregation. Such concentration-induced aggregation consequently causes a redshift. It should be noted that when the amount of $p$-phenylenediamine is increased up to $0.1 \mathrm{~g}$, the obtained samples show a dark red color due to serious aggregation, while the fluorescence suffers severe quenching. When the amount of $o$-phenylenediamine is increased up to $0.1 \mathrm{~g}$, the fluorescence still exhibits a yellow color, confirming that the red fluorescence is closely linked to the starting material (as seen in Fig. S4†). Furthermore, it is interesting to find that the emission peaks of these CDs clearly show a red-shift when dispersed in water. As shown in Fig. 4, the PL peaks of O-CDs $(0.005 \mathrm{~g}, 0.01 \mathrm{~g}, 0.05 \mathrm{~g})$ can shift from $520 \mathrm{~nm}, 530 \mathrm{~nm}, 550 \mathrm{~nm}$ to $540 \mathrm{~nm}, 550 \mathrm{~nm}$ and $580 \mathrm{~nm}$, while, the PL peak of P-CDs $(0.005 \mathrm{~g}, 0.01 \mathrm{~g}, 0.05 \mathrm{~g})$ shift from $575 \mathrm{~nm}, 580 \mathrm{~nm}, 610 \mathrm{~nm}$ to $600 \mathrm{~nm}, 608 \mathrm{~nm}$ and $630 \mathrm{~nm}$, respectively. The color of the samples also changes visibly (inset in Fig. 4). This solvent-dependent phenomenon is very similar to the "solvatochromism" in organic dyes. This effect is common for organic dyes, but is rarely known in CDs. ${ }^{29}$ This solvent-dependent fluorescence is regarded as "dipole induced surface electronic state change". According to XPS data, these CDs have abundant $\mathrm{N}$ and $\mathrm{O}$ elements. In addition, many $\mathrm{N}$ atoms are bonded to the edge of the $\mathrm{sp}^{2}$-hybridized carbon core in their pyridine and pyrrole forms. The $\mathrm{N}$ and $\mathrm{O}$ elements present in the respective structures enhance the charge carrier 

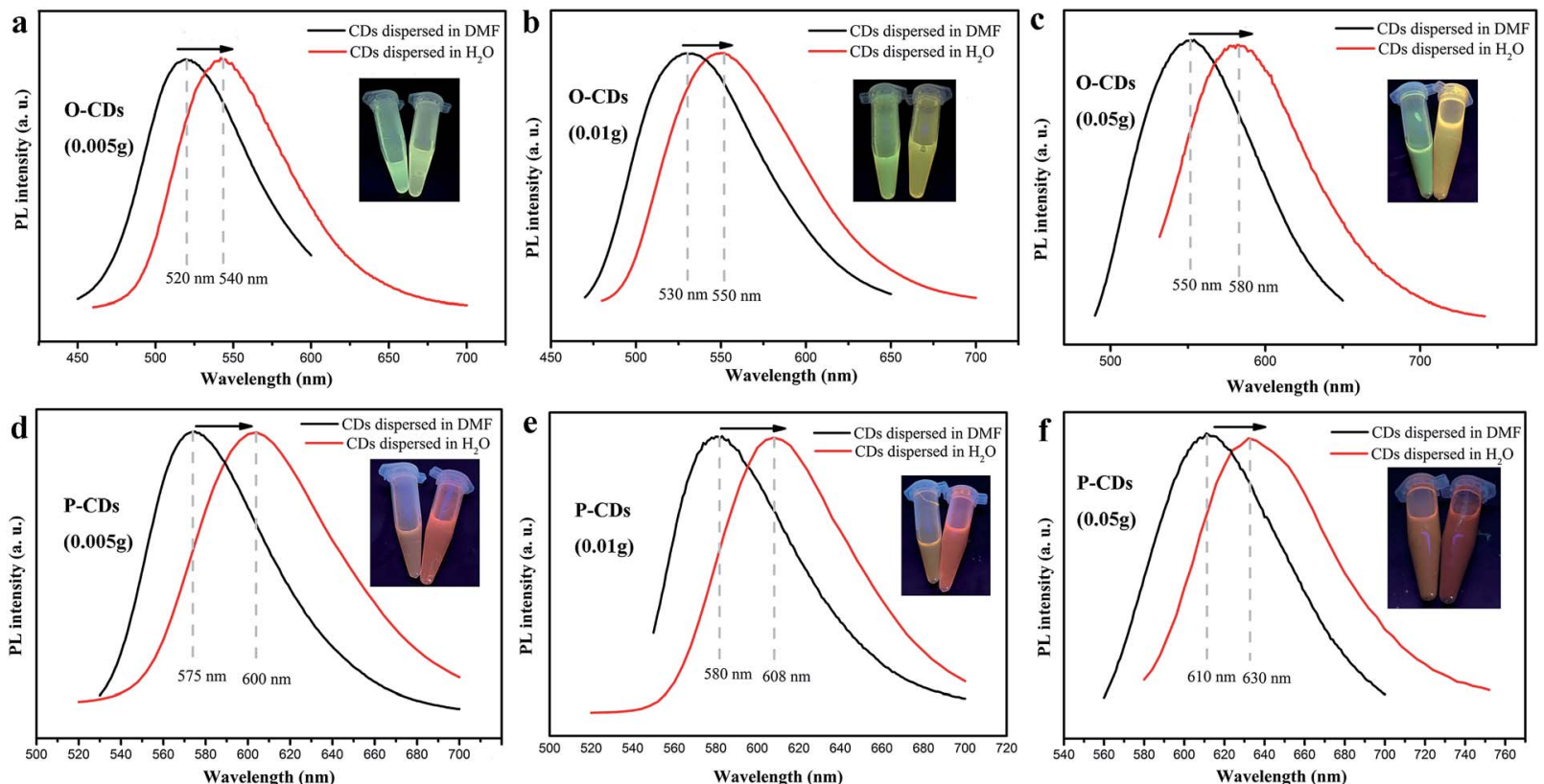

Fig. 4 The PL spectra of (a) O-CDs-0.005, (b) O-CDs-0.01 (c) O-CDs, (d) P-CDs-0.005, (e) P-CDs-0.01 and (f) P-CDs dispersed in DMF (black line) and $\mathrm{H}_{2} \mathrm{O}$ (red line) solution. The insets are the samples under UV light.

density and induce the charge transfer of electrons towards the edge. As the solvent polarity increases, the dipole moment significantly affects the surface electronic structure. Further, it reduces the energy gap, resulting in a red shift of fluorescence emission wavelength. ${ }^{30,31}$ Thus, we could acquire tunable CDs through adjusting starting materials, their amounts as well as the solvent. The QY of O-CDs and P-CDs in their aqueous solutions was also investigated and noted in Table S1.† Accompanying with evident red-shift, the fluorescence of these solutions had lowered slightly. Finally, the $\mathrm{pH}$ of the P-CDs and O-CDs solutions also affected the PL properties (Fig. S5†); significantly strong optical responses were observed when the $\mathrm{pH}$ of the solutions was varied. Under a strong acid solution $(\mathrm{HCl}, \mathrm{pH}=2)$, the PL quenched rapidly, but further on addition of a strong alkaline solution, such as $\mathrm{NaOH}$ solution $(\mathrm{pH}=14)$, the quenched CDs recovered their fluorescence instantaneously.

Although the mechanism for long-wavelength emission from the CDs is unclear, the amazing PL of CDs continues to motivate researchers to conduct deeper explorations. In general, researchers believe that the large size of particles and high nitrogen content are responsible for the efficient long-wavelength emission. ${ }^{27}$ Through careful examination of the characterization data such as those of TEM, XPS and FTIR, it was observed that the particle size and nitrogen content of the two CDs were found to be distinct. This suggests that a conjugate effect or quantum-size effect ${ }^{19}$ and higher nitrogen content, could be $t$ partly responsible for the red emission of the P-CDs. However, further in-depth studies are required to address this issue.

It is well known that fluorescence is often quenched when the aqueous dispersion of CDs are dried because of the direct $\pi-\pi$ interactions, which is due to a major Förster resonance energy transfer (FRET) process that is observed in aggregated
CDs. Hence, it is high time to establish an effective method to preserve the PL abilities of CDs in solid-state. In this study, PVB was found to be the best material for fabricating solid-states CDs such as CDs film (CF). The reasons are as follows:

(1) PVB possesses large chain structures that can keep appropriate distance between each other, so that the solid quenching can be restrained.

(2) PVB could be well compatible with CDs because of the abundant hydrophilic groups. Therefore, CDs can be homogeneously dispersed in PVB to avoid aggregation, which overcomes poor dispersion of CDs in a traditional curing agent. $\mathbf{a}$

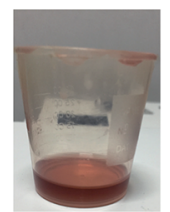

b
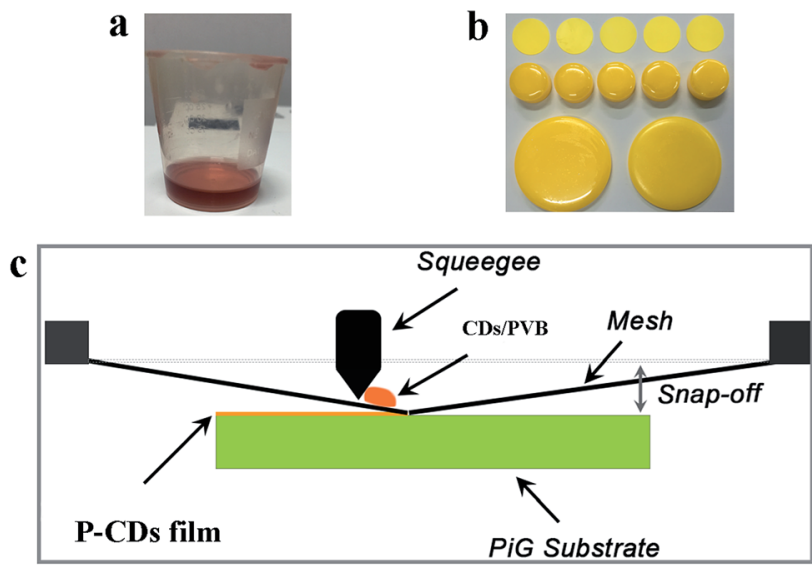

Fig. 5 (a) The composites of combined CDs solution and PVB in ethanol; (b) the sample of Ce:YAG PiG; (c) the schematic of fabrication process of red CDs stacked PiG (PiG-CF) plate via screen-printing technology. 
(3) PVB neither competes for UV light with CDs nor absorbs the emission obtained from CDs; the optical properties of CDs can remain unaffected after solidification.

(4) PVB powders readily dissolve in solutions at room temperature, which provides a facile and convenient method to fabricate films in comparison to other polymers such as PVA, and epoxy resin. As presented in Fig. 5a and b, tunable colors, tunable thickness, and tunable shapes could be easily acquired. Furthermore, the PL property of CF is depicted in Fig. S6. $\dagger$ It is observed that the solid state CDs continue to exhibit bright fluorescence after storage in air for two months, indicating their excellent stability. Moreover, the PL stability of these CFs in acidic solutions was tested under $1 \mathrm{~h}$ of UV irradiation, and almost no PL attenuation was observed (Fig. S7†). Compared with CDs solutions in acidic medium, PVB can prevent the quenching of PL of CDs, and the improved acid-resistance behavior of CFs also demonstrates that solid-state CFs are superior to CDs solution in several potential applications.

To explain the phenomenon, a mechanism of PL selfquenching in solid state CDs and the resistance against solidstate quenching of CDs embedded into PVB are illustrated in Fig. S8. $\dagger$ The TEM result shows CDs particles dispersed homogeneously in solution, which leads to the large separation among individual CDs. Hence, the CDs solutions always exhibit intense PL. However, the distance among individual CDs on drying is shortened to less than $R_{\mathrm{O}}$ ( $R_{\mathrm{O}}$ : Förster distance), and the FRET eventually leads to PL self-quenching. In CDs/PVB composites, the distance among individual CDs is embedded into the long chain of PVB. Therefore, individual CDs can keep an appropriate distance between each other, avoiding the FRET and consequently the solid-state CDs continue to exhibit bright PL.

\section{Application for warm WLED}

To fabricate warm WLEDs, screen-printing technology was adopted to fabricate PiG-CF composite material (the schematic depicted in Fig. 5). The $\mathrm{TeO}_{2}-\mathrm{ZnO}-\mathrm{Sb}_{2} \mathrm{O}_{3}-\mathrm{Al}_{2} \mathrm{O}_{3}-\mathrm{B}_{2} \mathrm{O}_{3}-\mathrm{Na}_{2} \mathrm{O}$ glass system was chosen as the optimal glass matrix for embedding $\mathrm{Ce}^{3+}$ :YAG phosphor, as previously reported by our group. ${ }^{32}$ The related PL and PL excitation (PLE) exhibited by PiG-CF composite is shown in Fig. S9. $\dagger$ As demonstrated in Fig. $6 \mathrm{c}$ and d, the electroluminescence (EL) spectra of the constructed WLEDs, presents an emission band peaking at $460 \mathrm{~nm}$ from the GaN chip and a broad yellow emission band from PiG. A different form of pure PiG (Fig. 6c) and PiG-CF (Fig. 6d) exhibits an obvious red emission band, which is attributed to the red CDs. As shown in Fig. 6e, the color coordinates of CIE shifted from $(0.3399,0.3594)$ to $(0.3943,0.3869)$, the CCT is reduced from $5220 \mathrm{~K}$ to $3722 \mathrm{~K}$, LE decreased from $122.1 \mathrm{~lm}$ $\mathrm{W}^{-1}$ to $66.17 \mathrm{~lm} \mathrm{~W}^{-1}$, and the CRI increased from 75.2 to 83 . The related photoelectric parameters are tabulated in detail in

Table 3 Photoelectric parameters of warm white LEDs under the operation current of $20 \mathrm{~mA}$

\begin{tabular}{lllll}
\hline Sample & CCT $(\mathrm{K})$ & CRI & LE $\left(\operatorname{lm~W}^{-1}\right)$ & Chromaticity coordinates \\
\hline PiG & 5220 & 75.2 & 122.1 & $(0.3399,0.3594)$ \\
PiG-CF & 3722 & 83 & 66.17 & $(0.3943,0.3869)$
\end{tabular}

$\mathbf{a}$
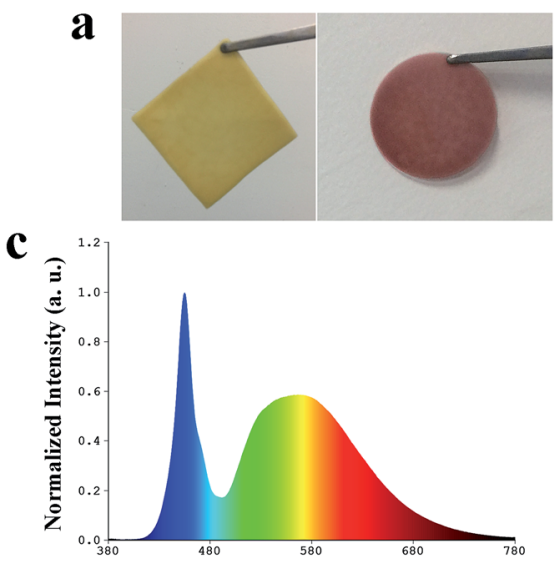

d

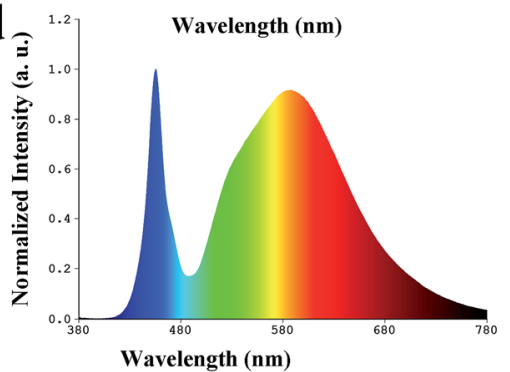

b

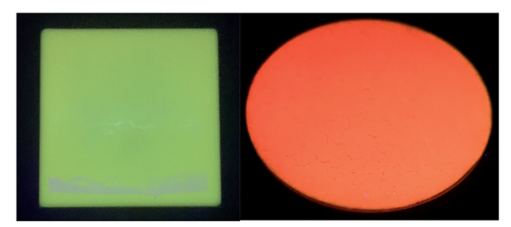

e

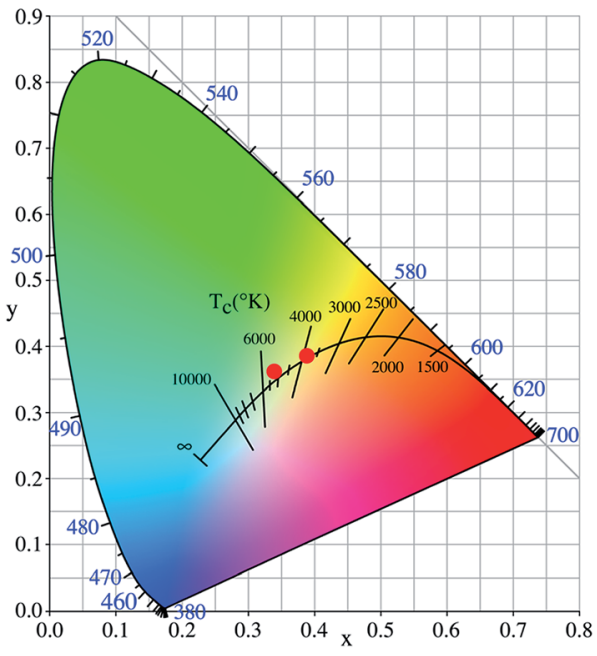

Fig. 6 (a and b) PCF, OCF under sunlight and UV light; (c and d) the normalized EL spectra for the pure PiG and PiG-CF; (e) CIE color coordinates of the warm white LEDs fabricated by coupling the $460 \mathrm{~nm}$ blue light with PiG and PiG-CF. 
Table 3. These results demonstrated that the CDs have potential application in in-door lighting.

\section{Conclusion}

In summary, multicolor CDs were synthesized via hydrothermal method using $p$-phenylenediamine or $o$-phenylenediamine as a precursor and DMF as the solvent. Moreover, O-CDs and P-CDs show a high QY of $58.8 \%$ and $52.4 \%$, respectively. These CDs exhibited different fluorescence emission colors when the amount of the carbon source was altered or when the CDs were dispersed in different solvents. In addition, the CDs solutions exhibited varying optical responses on adjusting the $\mathrm{pH}$ of solutions. The PL intensity rapidly decreased in acidic conditions, while it recovered immediately after adding an alkaline solution. Impressively, a series of tunable colors, tunable thickness and tunable shape CDs based solid-state materials could be easily acquired via mixing PVB and CDs. Finally, screen-printing technology was used to fabricate PiG-CF composite materials that could be employed to construct warm WLEDs on combining them with GaN chips.

\section{Conflicts of interest}

There are no conflicts to declare.

\section{Acknowledgements}

The authors are thankful to the National Nature Science Foundation of China (No. 51472183 and 51672192).

\section{References}

1 S. Zhu, Q. Meng, L. Wang, J. Zhang, Y. Song, H. Jin, K. Zhang, H. Sun and H. Wang, Highly photoluminescent carbon dots for multicolor patterning, sensors, and bioimaging, Angew. Chem., Int. Ed., 2013, 52, 3953-39537.

2 Y. P. Sun, B. Zhou, Y. Lin, W. Wang, K. A. S. Fernando, P. Pathak, M. J. Meziani, B. A. Harruff, X. Wang, P. G. Luo, H. Yang, M. E. Kose, B. Chen, L. M. Veca and S. Y. Xie, Quantum-Sized Carbon Dots for Bright and Colorful Photoluminescence, J. Am. Chem. Soc., 2006, 128, 7756-7757.

3 W. Shi, Q. Wang, Y. Long, Z. Cheng, S. Chen, H. Zheng and Y. Huang, Carbon nanodots as peroxidase mimetics and their applications to glucose detection, Chem. Commun., 2011, 47, 6695-6697.

4 K. Hola, Y. Zhang, Y. Wang, E. P. Giannelis, R. Zboril and A. L. Rogach, Carbon dots-emerging light emitters for bioimaging, cancer therapy and optoelectronics, Nano Today, 2014, 9, 590-603.

5 X. Li, Y. Liu, X. Song, H. Wang, H. Gu and H. Zeng, Intercrossed carbon nanorings with pure surface states as low-cost and environment-friendly phosphors for whitelight-emitting diodes, Angew. Chem., 2015, 54, 1759-1764.

6 H. Tetsuka, R. Asahi, A. Nagoya, K. Okamoto, I. Tajima, R. Ohta and A. Okamoto, Optically tunable amino- functionalized graphene quantum dots, Adv. Mater., 2012, 24, 5333-5338.

7 S. Zhu, Y. Song, X. Zhao, J. Shao, J. Zhang and B. Yang, The photoluminescence mechanism in carbon dots (graphene quantum dots, carbon nanodots, and polymer dots): current state and future perspective, Nano Res., 2015, 8, 355-381.

8 Y. Zhang, Y. Wang, X. Feng, F. Zhang, Y. Yang and X. Liu, Effect of reaction temperature on structure and fluorescence properties of nitrogen-doped carbon dots, Appl. Surf. Sci., 2016, 387, 1236-1246.

9 S. J. Zhu, J. H. Zhang, C. Y. Qiao, S. J. Tang, Y. F. Li, W. J. Yuan, B. Li, L. Tian, F. Liu, R. Hu, H. N. Gao, H. T. Wei, H. Zhang, H. C. Sun and B. Yang, Strongly green-photoluminescent graphene quantum dots for bioimaging applications, Chem. Commun., 2011, 47, 68586860.

10 Y. Q. Dong, J. W. Shao, C. Q. Chen, H. Li, R. X. Wang, Y. W. Chi, X. M. Lin and G. N. Chen, Blue luminescent graphene quantum dots and graphene oxide prepared by tuning the carbonization degree of citric acid, Carbon, 2012, 50, 4738-4743.

11 W. X. Zhang, D. J. Dai, X. F. Chen, X. X. Guo and J. Y. Fan, Red shift in the photoluminescence of colloidal carbon quantum dots induced by photon reabsorption, Appl. Phys. Lett., 2014, 104, 091902.

12 S. N. Qu, X. Y. Liu, X. Y. Guo, M. H. Chu, L. G. Zhang and D. Z. Shen, Amplified Spontaneous Green Emission and Lasing Emission from Carbon Nanoparticles, Adv. Funct. Mater., 2014, 24, 2689-26095.

13 H. Nie, M. J. Li, Q. S. Li, S. J. Liang, Y. Y. Tan, L. Sheng, W. Shi and S. X. A. Zhang, Carbon Dots with Continuously Tunable Full-Color Emission and Their Application in Ratiometric pH Sensing, Chem. Mater., 2014, 26, 3104-3112.

$14 \mathrm{~S} . \mathrm{Hu}, \mathrm{A}$. Trinchi, P. Atkin and I. Cole, Tunable Photoluminescence Across the Entire Visible Spectrum from Carbon Dots Excited by White Light, Angew. Chem., 2015, 54, 2970-2974.

15 H. M. Zhu, C. C. Lin, W. Q. Luo, S. T. Shu, Z. G. Liu, Y. S. Liu, J. T. Kong, E. Ma, Y. G. Cao, R. S. Liu and X. Y. Chen, Highly efficient non-rare-earth red emitting phosphor for warm white light-emitting diodes, Nat. Commun., 2014, 5, 4312.

16 D. Q. Chen, W. D. Xiang, X. J. Liang, J. S. Zhong, H. Yu, M. Y. Ding, H. W. Lu and Z. G. Ji, Advances in transparent glass-ceramic phosphors for white light-emitting diodes-A review, J. Eur. Ceram. Soc., 2015, 35, 859-869.

17 L. Bao, C. Liu, Z. Zhang and D. Pang, PhotoluminescenceTunable Carbon Nanodots: Surface-State Energy-Gap Tuning, Adv. Mater., 2015, 27, 1663-1667.

18 F. Yuan, Z. Wang, X. Li, Y. Li, Z. Tan, L. Fan and S. Yang, Bright Multicolor Bandgap Fluorescent Carbon Quantum Dots for Electroluminescent Light-Emitting Diodes, Adv. Mater., 2017, 29, 1604436.

19 K. Jiang, S. Sun, L. Zhang, Y. Lu, A. Wu, C. Cai and H. Lin, Red, Green, and Blue Luminescence by Carbon Dots: FullColor Emission Tuning and Multicolor Cellular Imaging, Angew. Chem., Int. Ed., 2015, 54, 5360-5364. 
20 X. Bai, C. GGianvito, Z. Hao, V. T. Freitas, J. Zhang, R. L. Longo, O. L. Malta, R. A. S. Ferreira and N. Pinna, Efficient and tuneable photoluminescent boehmite hybrid nanoplates lacking metal activator centres for single-phase white LEDs, Nat. Commun., 2014, 5, 5702.

21 S. K. Bhunia, S. Nandi, R. Shiklerb and R. Jelinek, Tuneable light-emitting carbon-dot/polymer flexible films prepared through one-pot synthesis, Nanoscale, 2016, 8, 3400-3406.

22 S. Lin, Z. Wang, Y. Zhang, Y. Huang, R. Yuan, W. Xiang and Y. Zhou, Easy synthesis of silver nanoparticles-orange emissive carbon dots hybrids exhibiting enhanced fluorescence for white light emitting diodes, J. Alloys Compd., 2017, 700, 75-82.

23 S. Lin, M. Chen, Z. Wang, Y. Zhang, R. Yuan, X. Liang, W. Xiang and Y. Zhou, Construction of full-color lightemitting $\mathrm{N}$-based carbon nanodots and their efficient solid-state materials via tape-casting technology for warm WLED, Chem. Eng. J., 2017, 324, 194-202.

24 S. Qu, D. Zhou, D. Li, W. Ji, P. Jing, D. Han, L. Liu, H. Zeng and D. Shen, Toward efficient orange emissive carbon nanodots through conjugated $\mathrm{sp}^{2}$-domain controlling and surface charges engineering, Adv. Mater., 2016, 28, 35163521.

25 T. F. Yeh, C. Y. Teng, S. J. Chen and H. Teng, Nitrogen-Doped Graphene Oxide Quantum Dots as Photocatalysts for Overall Water-Splitting under Visible Light Illumination, $A d v$. Mater., 2014, 26, 3297-3303.
26 S. Sarkar, M. Sudolská, M. Dubecký, C. J. Reckmeier, A. L. Rogach, R. Zbořil and M. Otypepka, Graphitic nitrogen doping in carbon dots causes red-shifted absorption, J. Phys. Chem. C, 2015, 120, 1303-1308.

27 S. Qu, X. Wang, Q. Lu, X. Liu and L. Wang, A biocompatible fluorescent ink based on water-soluble luminescent carbon nanodots, Angew. Chem., 2012, 51, 12215-12218.

28 X. Meng, Q. Chang, C. Xue, J. Yang and S. Hu, Full-colour carbon dots: from energy-efficient synthesis of concentration-dependent photoluminescence properties, Chem. Commun., 2017, 53, 3074-3078.

29 H. Wang, C. Sun, X. Chen, Y. Zhang, V. L. Colvin, Q. Rice, J. Seo, S. Feng, S. Wang and W. W. Yu, Excitation wavelength independent visible color emission of carbon dots, Nanoscale, 2017, 9, 1909-1915.

30 F. Terenziani, A. Painelli, C. Katan, M. Charlot and M. B. Desce, Charge instability in quadrupolar chromophores: Symmetry breaking and solvatochromism, J. Am. Chem. Soc., 2016, 128, 15742-15755.

31 P. Lekha and E. Prasad, Tunable emission of state excimer in a pyrene-modified polyamidoamine dendrimer aggregate through positive solvatochromism, Chem.-Eur. J., 2011, 31, 8609-8617.

32 J. Huang, X. Hu, J. Shen, D. Wu, C. Yin, W. Xiang and $\mathrm{X}$. Liang, Facile synthesis of thermally stable $\mathrm{Ce}^{3+}: \mathrm{Y}_{3} \mathrm{Al}_{5} \mathrm{O}_{12}$ phosphor-in-glass for white LEDs, CrystEngComm, 2015, 17, 7079-7085. 